\title{
Arte y Diseño Discursos de la Identidad Cultural en América Latina Proyecto de Investigación \\ $\mathrm{N}^{\circ} 11.3$ \\ Equipo de Investigación ${ }^{(1)}$ \\ Natalia Aguerre \\ Cynthia Lizette Hurtado Espinosa \\ por Universidad de Guadalajara (MEX)
}

por Facultad de Diseño y Comunicación, Universidad de Palermo (ARG),

\begin{abstract}
Resumen: El Proyecto de Investigación 11.3 reflexiona sobre las prácticas artísticas y de diseño en tanto representaciones de identidad cultural de la comunidad desde donde emergen. Esta temática conlleva a problematizar el discurso modernista dominante, el que otorgaba a las representaciones de "lo latinoamericano" características que han permanecido encasilladas en lo mágico, surreal o fantástico rechazando aquellas manifestaciones que, a partir de sus expresiones diversas y diferenciales con los cánones europeos en su concepción moderna. La producción y creación estética de la cultura en nuestro territorio aporta valiosos insumos para que el pueblo se reconozca en su identidad, consolide su memoria y aporte su inteligencia sensible frente a preguntas sobre quiénes somos los latinoamericanos en esta transición milenaria, cuál es nuestro lugar en el mundo y cómo seguiremos enfrentando la vertiginosa globalización, cada día más acelerada por la telemática, la cibernética y la informática.
\end{abstract}

Palabras clave: Arte - Diseño - Identidad

[Resúmenes en inglés y portugués en la página 102]

${ }^{(1)}$ Los CVs del Equipo de Investigación pueden consultarse en el Capítulo Directores de Líneas y Coordinadores de Proyectos de esta misma Edición. 


\section{Acerca del Proyecto 11.3}

\section{Arte y Diseño \\ Discursos de la Identidad Cultural en América Latina}

El Proyecto 11.3 Arte y Diseño Discursos de la Identidad Cultural en América Latina, aborda el tema de la identidad cultural en América Latina desde una mirada estética comunicacional, donde se reflexiona sobre las prácticas artísticas y de diseño, que reivindica la producción y creación estética de la cultura de nuestro territorio, la que aporta valiosos insumos para que los pueblos se reconozcan en su identidad y consoliden su memoria.

Se acordó entre la Universidad de Palermo y la Universidad de Guadalajara (México), avanzar en una investigación en la que participan académicos de ambas Instituciones, con la coordinación compartida de Natalia Aguerre (UP) y Cynthia Lizette Hurtado Espinosa (Universidad de Guadalajara, México), con el fin de generar un corpus sólido de conocimientos sobre las representaciones de la identidad cultural de la región.

Las reflexiones y los resultados obtenidos en el Proyecto 11.3 Arte y Diseño Discursos de la Identidad Cultural en América Latina, son continuación de los Proyectos 11.2 Arte, Historia y Memoria y 11.1 Experiencias del Arte Latinoamericano y su incidencia sobre el tiempo y guardan relación con el Proyecto 11.4 Imagen e Identidad Política en América Latina en el marco de la misma Línea de Investigación y bajo la misma Directora Natalia Aguerre.

Sus principales objetivos son:

- Generar un espacio de participación crítica y producción de conocimiento, en torno a los procesos y prácticas artísticas desde la perspectiva comunicacional.

- Establecer el lugar político, social, cultural y económico de los análisis estéticocomunicacionales.

- Reflexionar sobre las prácticas artísticas y de diseño, entendidas como representaciones de la identidad cultural de América Latina.

- Cuestionar el discurso que otorga a "lo latinoamericano" características que aún parecen seguir encasilladas en lo mágico, surreal y/o fantástico.

- Reivindicar la producción y creación estética de la cultura de la región, a partir del aporte de sus valiosos insumos identitarios.

- Consolidar el espacio de la memoria e inteligencia sensible acerca de "quiénes somos los latinoamericanos", "cuál es nuestro lugar en el mundo" y "cómo seguiremos enfrentando la vertiginosa globalización".

\section{Acerca de la Línea 11}

Arte y Comunicación dirigida por Natalia Aguerre se desarrolla de manera ininterrumpida desde 2015 en la Facultad de Diseño y Comunicación (UP, Argentina), e incluye hasta el momento cuatro proyectos finalizados el 11.1 Experiencias del Arte Latinoamericano y su incidencia sobre el tiempo coordinado por Natalia Aguerre (UP, Argentina) y Marie 
Boivent (Universite Rennes 2, Francia), el 11.2 Arte, Historia y Memoria coordinado por Natalia Aguerre (UP, Argentina) y Carlos Paz (Universidade do Vale dos Sinos, Unisinos, Rio Grande do Sul, Brasil), el 11.3 Arte y Diseño: Discursos de la Identidad Cultural en América Latina coordinado por Natalia Aguerre (UP, Argentina) y Cynthia Lizette Hurtado Espinosa (Universidad de Guadalajara, México), y el 11.4 Imagen e Identidad Política en América Latina coordinado por Marina Mendoza por (UP, Conicet IEALC UBA, Argentina), Mariano Dagatti (Conicet, Universidad de Quilmes, Argentina) y Paulo Carlos López López (Universidad de Santiago de Compostela, España).

\section{Mapa de Áreas y Proyectos}

El Proyecto 11.3 se vincula con todas las carreras de la Facultad de Diseño y Comunicación puesto que los lineamientos abordados son transversales a los contenidos relacionados con el diseño en sus múltiples manifestaciones, y particularmente con la carrera de Arte Multimedial. Asimismo, sus resultados tienen una capacidad de impacto en las carreras de posgrado que exploran las interrelaciones entre arte, comunicación y diseño en toda la región.

\section{Productos y Resultados}

\section{a)- Publicaciones}

Cuaderno del Centro de Estudios de Diseño y Comunicación №101. (2021) Arte y Comunicación: Arte y Diseño: Discursos de la Identidad Cultural en América Latina. Coordinación Natalia Aguerre, Cynthia Lizette Hurtado Espinosa. Buenos Aires, Argentina. ISSN: 1668-0227.

Esta publicación documenta y comunica los resultados alcanzados en el proyecto de investigación 11.3 Arte y Diseño: Discursos de la Identidad Cultural en América Latina, y a continuación se detallan los autores y artículos contenidos en ella:

Aguerre Natalia, Lizette Cynthia Espinosa Hurtado (2021) Prólogo (Pp. 11-16) Campos Barragán Mariana Noemí, Hurtado Espinosa Cynthia Lizette, Casillas López Miguel Angel (2021) La identidad de los equipos de fútbol mexicanos a través de sus identificadores gráficos y su influencia en la cultura mexicana (Pp. 17-46)

Cisneros Hernández Adrián Antonio, Ramírez Mercado Marcela del Rocío, Olivares Gallo Juan Ernesto Alejandro (2021) La identidad en el turismo religioso de San Juan de los Lagos (Pp. 47-67)

Durán Alfaro Verónica, González Arce Jorge Alberto, Peña Mercado (2021) La identidad como eje integrador de una marca ciudad (Pp. 69-89)

Galindo Flores Eduardo, González Castañeda Mónica, Rodríguez Medina Daniel (2021) La gráfica popular, un referente de la identidad del diseño gráfico mexicano (Pp. 91-105) 
Galletti, Patricia Cecilia (2021) El flamenco en los Gitanos Calé: apuntes para una investigación sobre la innovación y la inclusión socioculturales desde la antropología y el diseño (Pp. 107-116)

García Hernández Amalia, Gutiérrez Cruz Irma Lucía, Osuna Ruiz Eva Guadalupe (2021) El alcance de la gastronomía mexicana en otras fronteras a través del diseño gráfico por el medio de la Web (Pp. 117-136)

Guardia Manzur, Alejandra (2021) A través del ojo colonial. Discursos visuales de la mujer indígena boliviana (Pp. 137-154)

Luna Abundis José Antonio, Gilberto Menchaca de Alba Noé, Polo Vázquez Nuño Marco (2021) La implementación del Video en proyectos de Diseño de Identidad Corporativa (Pp. 155-184)

Nava Le Favi, Daniela (2021) La práctica de vestir a la Mamita: legitimidades, identidades y arte popular en el caso de la Virgen de Urkupiña en la ciudad de Salta-Argentina (Pp. 185-195)

Samaniego Pesantez, Kléver Rolando (2021) Los macaneros y el diseño comunicacional de su organización (Pp. 197-212)

Tonatihu Hernández Salazar Agustín, Pérez Cortés María Eugenia (2021) El arte wixárika: un lenguaje visual para la defensa de su cultura (Pp. 213-234)

Mora Lomelí Leonardo, Orozco-Grover Gabriel, Santoyo Mercado Aurea (2021) Hecho en México para ojos extranjeros: uso y menosprecio de referentes identitarios nacionales en el diseño de productos de consumo (Pp. 235-257)

\section{b)- Congresos / Coloquios / Plenarios}

V Coloquio de Investigación y Desarrollo en Diseño Latino. Universidad de Palermo, 27 de julio de 2020. XI Congreso Latinoamericano de Enseñanza del Diseño. Semana Internacional del Diseño en Palermo. Se presentaron los resultados y avances de la Línea de Investigación №11 en dos comisiones Arte y Comunicación y Comunicación Política. En la comisión Arte y Comunicación, se presentaron las reflexiones y conclusiones del proyecto 11.3 Arte y Diseño: Discursos de la Identidad Cultural en América Latina La gráfica y los cinco elementos de identidad en torno al turismo religioso Verónica Durán Alfaro, Jorge Alberto González expusieron: Mariana Noemí Campos Barragán, Cynthia Lizette Hurtado Espinosa, Miguel Ángel Casillas López, Adrián Antonio Cisneros Hernández, Ramírez Mercado Marcela del Rocío, Juan Ernesto Alejandro Olivares Gallo, Verónica Durán Alfaro, Jorge Alberto González Arce, Claudia Mercado Peña, Eduardo Galindo Flores, Mónica González Castañeda y Daniel Rodríguez Medina, Patricia Cecilia Galletti, Amalia García Hernández, Irma Lucía Gutiérrez Cruz, Eva Guadalupe Osuna Ruiz, Alejandra Guardia Manzur, José Antonio Luna Abundis, Noé Gilberto Menchaca de Alba, Marco Polo Vázquez Nuño, Daniela Nava Le Favi, Kléver Rolando Samaniego Pesantez, Agustín Tonatihu Hernández Salazar María Eugenia Pérez Cortés, Leonardo Mora Lomelí, Aurea Santoyo Mercado y Gabriel Orozco-Grover. 
A continuación se detallan las ponencias presentadas en esta Comisión coordinada por la Directora del proyecto Natalia Aguerre:

Mariana Noemí Campos Barragán, Cynthia Lizette Hurtado Espinosa y Miguel Ángel Casillas López (México) Identidad gráfica de equipos representativos del balón-pie mexicano Adrián Antonio Cisneros Hernández, Ramírez Mercado Marcela del Rocío y Juan Ernesto Alejandro Olivares Gallo (México)

Arce y Claudia Mercado Peña (México)

La marca ciudad Guadalajara, su identidad e integración

Eduardo Galindo Flores, Mónica González Castañeda y Daniel Rodríguez Medina (México)

Memoria de la gráfica popular en México

Patricia Cecilia Galletti (Argentina)

El flamenco en los Gitanos Calé: apuntes para una investigación sobre la innovación y la inclusión sociocultural desde la antropología y el diseño

Amalia García Hernández, Irma Lucía Gutiérrez Cruz y Eva Guadalupe Osuna Ruiz (México)

El uso de la fotografía en el diseño gráfico, aplicado en los platillos gastronómicos mexicanos más representativos por medio de la Web

Alejandra Guardia Manzur (Argentina)

A través del ojo colonial. Discursos visuales de la mujer indígena boliviana

José Antonio Luna Abundis, Noé Gilberto Menchaca de Alba y Marco Polo Vázquez Nuño (México)

El brand reveal como un vehículo de comunicación estratégico para el diseño de identidad

Daniela Nava Le Favi (Argentina)

La práctica de vestir a la Mamita: legitimidades, identidades y arte popular, en el caso de la Virgen de Urkupiña en la ciudad de Salta-Argentina

Kléver Rolando Samaniego Pesantez (Ecuador)

Los macaneros y el diseño comunicacional de su organización

Agustín Tonatihu Hernández Salazar y María Eugenia Pérez Cortés (México)

Origen y razón del arte moderno Wixárika

Leonardo Mora Lomelí, Aurea Santoyo Mercado y Gabriel Orozco-Grover (México)

Identidad nacional, prejuicios y diseño: el uso de referentes gráficos mexicanos en la comercialización de productos de consumo

\section{c)- Formación de Posgrado e Impacto curricular}

La Directora Natalia Aguerre es Docente de Posgrado en Diseño de la Facultad de Diseño y Comunicación: Doctorado en Diseño y Maestría en Gestión del Diseño y al igual que Cynthia Lizette Hurtado Espinosa (Universidad de Guadalajara, México) en su institución, incorporan los contenidos de su investigación a sus asignaturas en posgrado. 


\begin{abstract}
Research Project 11.3 reflects on artistic and design practices as representations of the cultural identity of the community from which they emerge. This theme leads to problematize the dominant modernist discourse, which gave representations of "the Latin American" characteristics that have remained pigeonholed in the magical, surreal or fantastic, rejecting those manifestations that, based on their diverse and differential expressions with the European canons in its modern conception. The aesthetic production and creation of culture in our territory provides valuable inputs for the people to recognize their identity, consolidate their memory and contribute their sensitive intelligence to questions about who are Latin Americans in this millennial transition, what is our place in the world and how we will continue to face the dizzying globalization, increasingly accelerated by telematics, cybernetics and computing.
\end{abstract}

Keywords: Art - Design - Identity

Resumo: O Projeto de Pesquisa 11.3 reflete sobre as práticas artísticas e de design como representações da identidade cultural da comunidade da qual emergem. Esse tema leva a problematizar o discurso modernista dominante, que deu representações de características "latino-americanas" que permaneceram classificadas no mágico, surreal ou fantástico, rejeitando aquelas manifestações que, a partir de suas expressões diversas e diferenciadas com os cânones europeus em sua concepção moderna. A produção estética e a criação de cultura em nosso território fornecem valiosos insumos para que as pessoas reconheçam sua identidade, consolam sua memória e contribuam com sua inteligência sensível às questões sobre quem são os latino-americanos nesta transição milenar, qual é o nosso lugar no o mundo e como continuaremos a enfrentar a vertiginosa globalização, cada vez mais acelerada pela telemática, a cibernética e a tecnologia da informação.

Palavras chave: Arte - Design - Identidade

[Las traducciones de los resúmenes fueron realizadas a través de traductor automático] 